\title{
Single-nanowire solar cells beyond the Shockley-Queisser limit
}

\author{
Peter Krogstrup ${ }^{1 \dagger}$, Henrik Ingerslev Jørgensen ${ }^{2 \dagger}$, Martin Heiss ${ }^{3 \dagger}$, Olivier Demichel ${ }^{3}$, Jeppe V. Holm ${ }^{2}$, \\ Martin Aagesen², Jesper Nygard' and Anna Fontcuberta i Morral ${ }^{3 \star}$
}

\begin{abstract}
Light management is of great importance in photovoltaic cells, as it determines the fraction of incident light entering the device. An optimal p-n junction combined with optimal light absorption can lead to a solar cell efficiency above the Shockley-Queisser limit. Here, we show how this is possible by studying photocurrent generation for a single core-shell p-i-n junction GaAs nanowire solar cell grown on a silicon substrate. At 1 sun illumination, a short-circuit current of $180 \mathrm{~mA} \mathrm{~cm}^{-2}$ is obtained, which is more than one order of magnitude higher than that predicted from the Lambert-Beer law. The enhanced light absorption is shown to be due to a light-concentrating property of the standing nanowire, as shown by photocurrent maps of the device. The results imply new limits for the maximum efficiency obtainable with III-v based nanowire solar cells under 1 sun illumination.
\end{abstract}

N anowire-based solar cells hold great promise for third-generation photovoltaics and for powering nanoscale devices ${ }^{1,2}$. With the advent of third-generation photovoltaics, solar cells will become cheaper and more efficient than current devices. In particular, a cost reduction may be achieved by reducing material use through the fabrication of nanowire arrays and radial $\mathrm{p}-\mathrm{n}$ junctions $s^{3-5}$. The geometry of nanowire crystals is expected to favour elastic strain relaxation, providing great freedom in the design of new compositional multijunction solar cells ${ }^{6}$ grown on mismatched materials ${ }^{7,8}$. The efficiencies of nanostructured solar cells have increased over time and have now reached up to $13.8 \%$, due to improvements in materials and new device concepts ${ }^{9-14}$.

Light absorption in standing nanowires is a complex phenomenon, with a strong dependence on nanowire dimensions and the absorption coefficient of the raw materials ${ }^{15-18}$. In low-absorbing microwire arrays, such as those composed of silicon, light absorption is understood via ray optics or by calculation of the integrated local density of optical states of the nanowire film ${ }^{19,20}$. Interestingly, when these arrays stand on a Lambertian back-reflector, an asymptotic increase in light trapping for low filling factors (FFs) is predicted $^{19}$. This is advantageous for improvement of the efficiency-to-cost ratio of solar cells and has led to the demonstration of microwire arrays exhibiting higher absorption than in the equivalent thickness of textured film ${ }^{19,21,22}$. The case for nanowires is quite different. Nanowire diameters are smaller than or comparable to the radiation wavelength. In this case, optical interference and guiding effects play a dominant role in relation to reflectivity and absorption spectra. For low-absorbing materials (for example, indirect bandgap materials such as silicon), waveguiding effects plays a key role 23,24 , whereas highly absorbing semiconductors (such as direct-bandgap GaAs) exhibit resonances that increase the total absorption several times. Nanowires lying on a substrate also exhibit such resonances, often described by Mie theory ${ }^{25,26}$, although the total absorption rate is significantly lower ${ }^{27,28}$. Even though the optical absorption of nanowires arranged in an array has been shown to be far more complex than in thin films, nanowire vertical arrays currently seem to be the most reasonable device proposal. One elegant device consists of a single standing nanowire solar cell, contacted on top by a transparent electrode and at the bottom through the substrate. Although the characterization of single nanowires lying on a substrate is quite common, to date there have been no studies on single standing nanowires.

In this Article, we present experimental measurements on single GaAs nanowire solar cells, as grown on a silicon substrate, where the p-section is contacted through a highly doped substrate and the n-section through a transparent top contact (Fig. $1 \mathrm{a}-\mathrm{c})^{26}$. We find that light absorption in single standing nanowires is more than one order of magnitude more efficient than is predicted from the Lambert-Beer law. We show measurements on two devices. The first device (Fig. 1) exhibits a short-circuit current density of $180 \mathrm{~mA} \mathrm{~cm}^{-2}$ when normalized to the projected area. This leads to an apparent solar conversion efficiency of $40 \%$. The second device shows a short-circuit current of $173 \mathrm{~mA} \mathrm{~cm}^{-2}$ and an apparent efficiency of $28 \%$. The reason for these very high efficiencies is the mismatch between the absorption cross-section and the physical bounds of the nanowires, hinting at a very large absorption crosssection. This work represents a critical step towards the next generation of nanowire-based solar cells.

The current-voltage characteristics of the devices were measured in the dark and under AM 1.5G illumination. The experimental data for device 1 are shown in Fig. 1d. In the dark, the device exhibits typical diode behaviour, with an ideality factor of 2.6. Under illumination, the diode curve is shifted downwards as a consequence of photogeneration and separation of electron-hole pairs, giving a short-circuit current of $256 \mathrm{pA}$. The diameter of the nanowire is $425 \mathrm{~nm}$, corresponding to an apparent photogenerated current density of $180 \mathrm{~mA} \mathrm{~cm}^{-2}$. The open-circuit voltage $V_{\mathrm{OC}}$ and FF are $0.43 \mathrm{~V}$ and 0.52 , respectively, both of which should be improved by optimizing the resistivity, the thickness of the doped layers and surface passivation ${ }^{29}$. The generated power at the maximum power point is $57 \mathrm{pW}$, corresponding to $40 \mathrm{~mW} \mathrm{~cm}^{-2}$. Dividing the generated power density by the incident power density, the

\footnotetext{
${ }^{1}$ Center for Quantum Devices, Nano-Science Center, Niels Bohr Institute, University of Copenhagen, Universitetsparken 5, DK-2100 Copenhagen, Denmark, ${ }^{2}$ SunFlake A/S, Nano-Science Center, Universitetsparken 5, DK-2100 Copenhagen, Denmark, ${ }^{3}$ Laboratoire des Matériaux Semiconducteurs, Ecole Polytechnique Fédérale de Lausanne, 1015 Lausanne, Switzerland; †These authors contributed equally to this work. ^e-mail: anna.fontcuberta-morral@epfl.ch; krogstrup@fys.ku.dk
} 
a

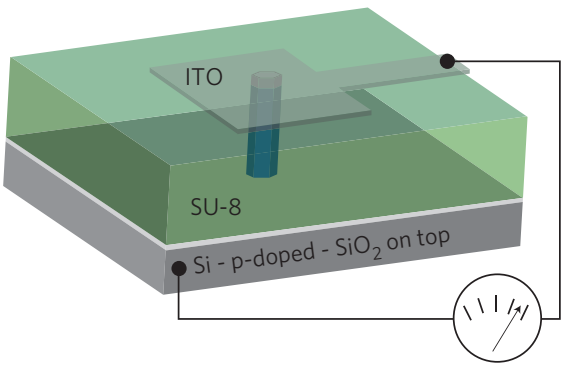

b
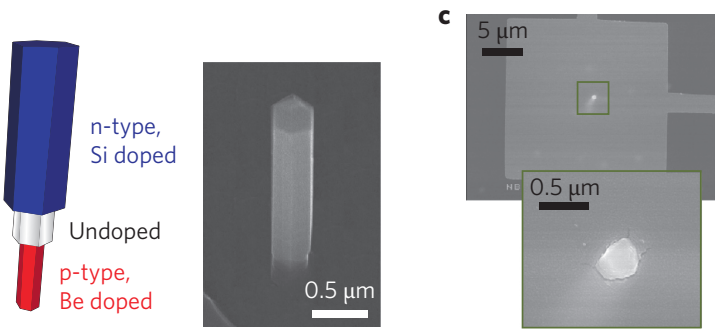

d

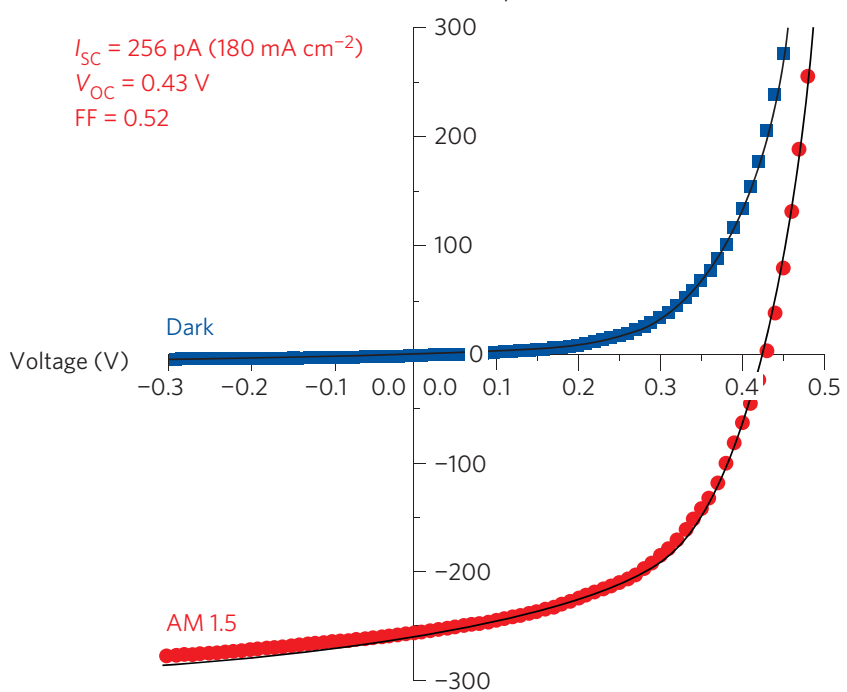

Figure 1 | Electrical characterization of a single nanowire solar cell (device 1). a, Schematic of the vertical single-nanowire radial $p-i-n$ device connected to a p-type doped silicon wafer by epitaxial growth. $\mathbf{b}$, Left: doping structure of the nanowire. The $p$-type doped core is in contact with the doped silicon substrate and the n-type doped shell is in contact with the ITO. Right: Scanning electron microscope (SEM) image of a nanowire solar cell before adding the top contact, with a $30^{\circ}$ angle from the vertical. c, SEM images of the device seen from the top electrode. The nanowire is $\sim 2.5 \mu \mathrm{m}$ high and has a diameter of $\sim 425 \mathrm{~nm}$. d, Current-voltage characteristics of the device in the dark and under AM 1.5G illumination, showing the figure-of-merit characteristics.

solar cell yields an apparent efficiency of $40 \%$. To understand the extreme photon collection boost in free-standing single GaAs nanowires, we used a finite-difference time-domain (FDTD) method to model a $2.5-\mu \mathrm{m}$-long nanowire embedded in SU-8 as a function of its diameter and of the wavelength of the plane-wave radiation propagating along the nanowire axis ${ }^{30-32}$. Figure $2 \mathrm{a}$ shows the wavelength and diameter dependence of the absorption rate of such a nanowire. Note that the absorption is zero for wavelengths larger than $900 \mathrm{~nm}$ where the absorption coefficient of GaAs goes to zero. Two dominant branches for low and high diameters are observed, corresponding to resonances similar to the Mie resonances observed in nanowires lying on a substrate ${ }^{25}$. Light absorption in the standing nanowire is enhanced by a factor of between 10 and 70 with respect to the equivalent thin film. Another way to express this enhancement in absorption is through the concept of an absorption cross-section. The absorption cross-section is defined as $A_{\mathrm{abs}}=a \eta$, where $a$ is the physical cross-section of the nanowire and $\eta$ is the absorption efficiency. It is largely accepted that the absorption cross-section in nanoscale materials is larger than their physical size. In systems such as quantum dots, the absorption cross-section can exceed the physical size by a factor of up to 8 (ref. 33). We calculated the absorption cross-section of the nanowires as a function of the nanowire diameter and incident wavelength (Fig. 2b). The absorption crosssection is, in all cases, larger than the physical cross-section of the nanowire. It is interesting to note that the absorption of photons from an area larger than the nanowire itself is equivalent to a builtin light concentration $C$. Light concentration has an additional benefit in that it increases the open-circuit voltage with a term $k T \ln C$, thereby increasing the efficiency ${ }^{34-36}$. The largest absorption cross-section in Fig. $2 \mathrm{~b}$ is $1.13 \times 10^{6} \mathrm{~nm}^{2}$ for a nanowire diameter of $380 \mathrm{~nm}\left(a=9.38 \times 10^{4} \mathrm{~nm}^{2}\right)$, corresponding to an overall builtin light concentration of $\sim 12$.

Measurements of the external quantum efficiency (EQE) normalized by the physical area for both lying and standing nanowire devices are shown in Fig. 3a (see Supplementary Section S1 for
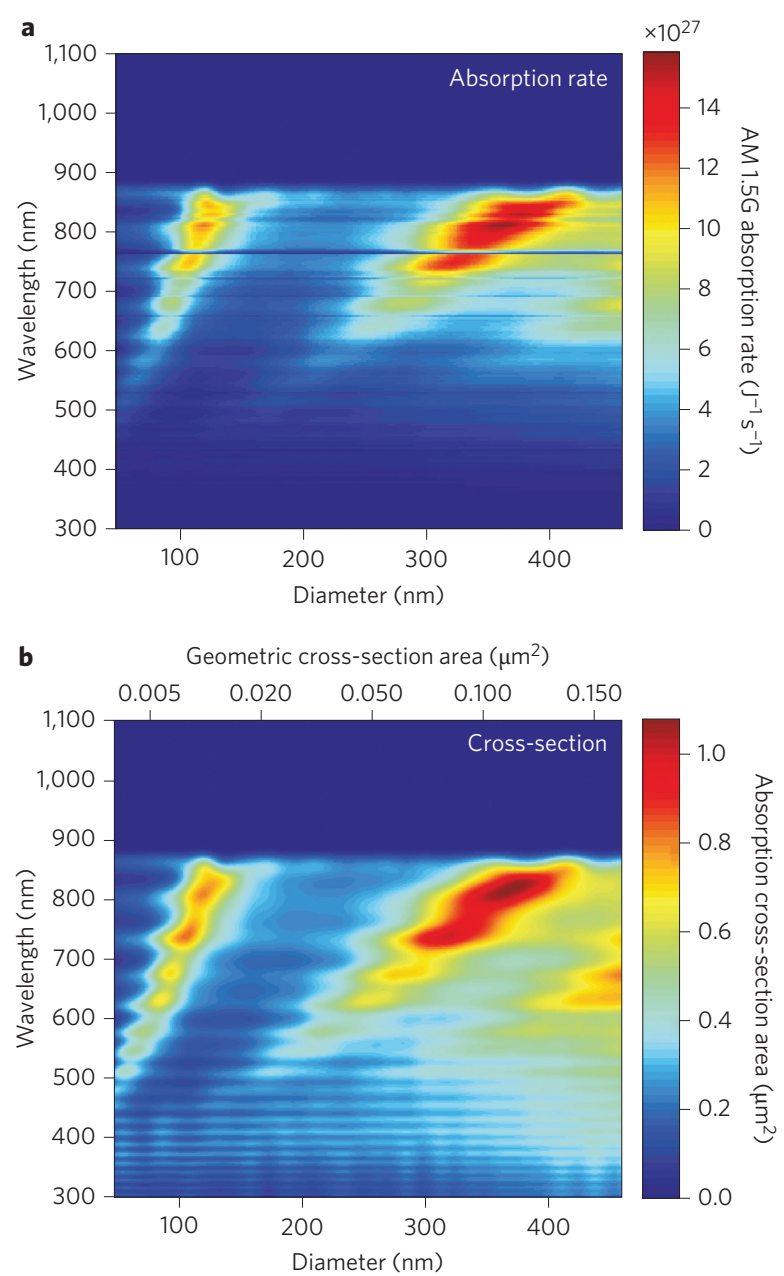

Figure 2 | Optical simulations of a single nanowire solar cell.

$\mathbf{a}, \mathbf{b}$, Simulations of light absorption in a $2.5 \mu \mathrm{m}$ standing GaAs nanowire that is fully embedded in SU-8 $(n=1.67)$ on a silicon substrate: the absorption rate of solar AM $1.5 \mathrm{G}$ radiation (a) and simulated absorption cross-section (b) exhibit two main resonant branches, similar to Mie resonances observed in nanowires lying on a substrate. The periodic modulation with wavelength is a result of Fabry-Pérot interference in the polymer layer and not an artefact of the simulation. 

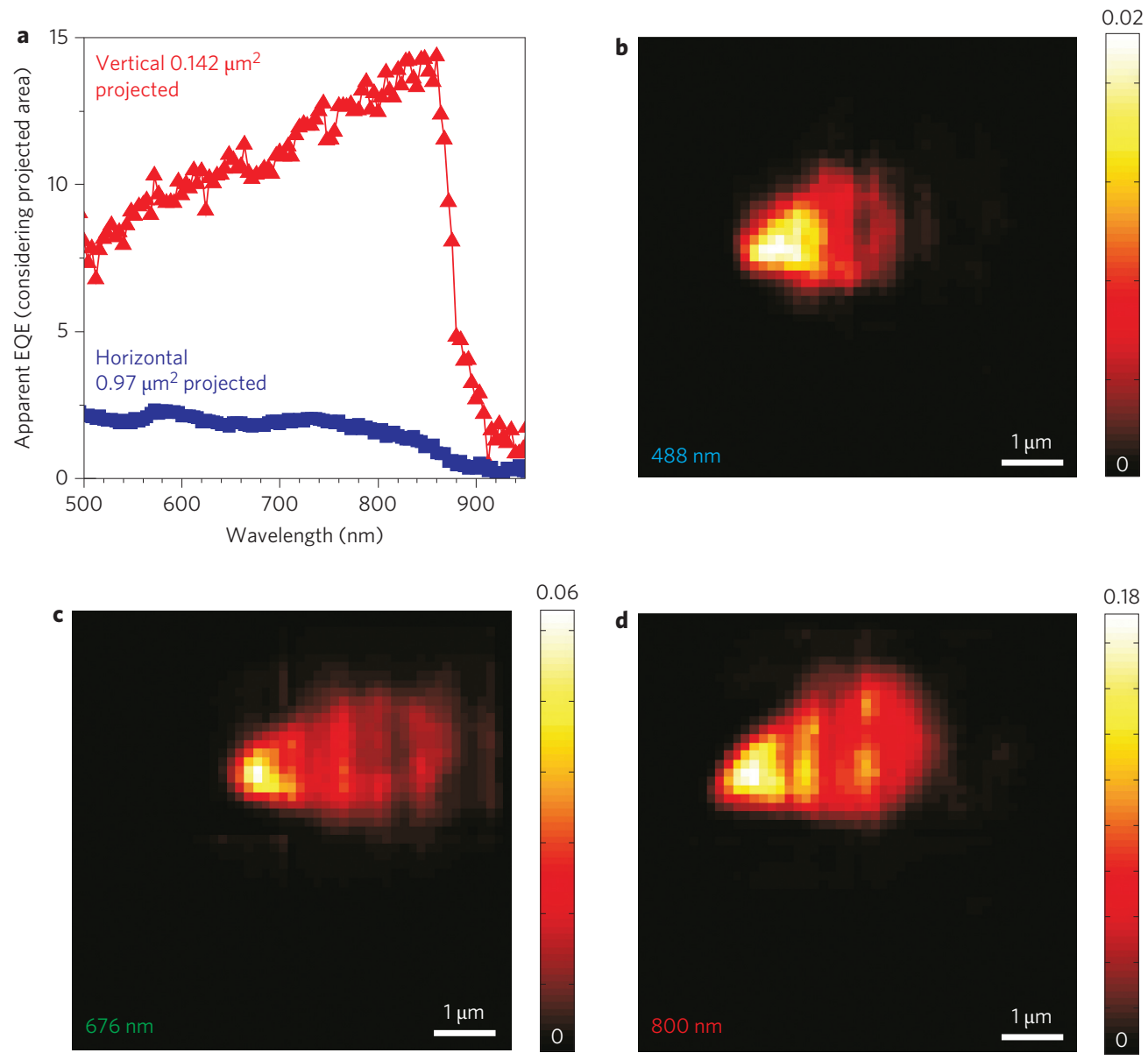

Figure 3 | Optical characterization of a single nanowire solar cell (device 2). a, EQE normalized by indicated projected area, comparing vertical and horizontal nanowire solar cells. For the vertical standing solar cell, a 15-fold increase in photon collection is obtained close to the bandgap. EQE becomes negligible for photon wavelengths below the bandgap of GaAs, meaning that there is no contribution from absorption in the silicon substrate ${ }^{45}$. b-d, Scanning photocurrent measurements on our single vertical nanowire device for three different excitation laser wavelengths, normalized to the incident photon flux. Scale bar, $1 \mu \mathrm{m}$

more details). Lying nanowires exhibit EQE values up to 2 due to Mie resonances ${ }^{37}$, while for standing nanowires values of up to $\sim 14.5$ are reached. This further confirms that the absorption cross-section is several times larger than the apparent crosssection of the wire, especially at wavelengths close to the bandgap.

To further understand the absorption boost in our devices, we spatially mapped the photocurrent generated by a vertical nanowire device for three different excitation wavelengths: $488 \mathrm{~nm}, 676 \mathrm{~nm}$ and $800 \mathrm{~nm}$. The results presented in Fig. $3 \mathrm{~b}-\mathrm{d}$ are deconvoluted with the point-spread function of the diffraction-limited laser spot. As seen in the figure, a photocurrent from an area much larger than the size of the laser spot appears for all three wavelengths. A fit to the data allows the estimation of an effective absorption crosssection diameter of $1.2 \mu \mathrm{m}(488 \mathrm{~nm}), 1.0 \mu \mathrm{m}(676 \mathrm{~nm})$ and $1.3 \mu \mathrm{m}$ $(800 \mathrm{~nm})$, respectively. Hence, the absorption boost in our device is due to an unexpectedly large absorption cross-section of the vertical nanowire geometry. This is equivalent to a built-in light concentration of $\sim 8$, which is in good agreement with our theoretical predictions. In addition, we speculate that the top-contact geometry further contributes to the resonant absorption effect, thereby increasing the absorption cross-section and the boost in photogenerated current.

Finally, we put our results into perspective by comparing them to the best existing technologies and to the design principles for increasing efficiency. In Table 1 we list the best values for leading technologies such as single-junction crystalline silicon (c-Si) and $\mathrm{GaAs}$, as well as triple-junction devices. The highest efficiency is provided by the triple-junction solar cell (34.1\%), with a shortcircuit current of $14.7 \mathrm{~mA} \mathrm{~cm}^{-2}$. In this case the short-circuit current is kept relatively low as it has to be matched between the three cells connected in series, and it is the high efficiency that generates the increase in $V_{\mathrm{OC}}$. The highest short-circuit current is obtained in the c-Si solar cell, where light management techniques result in a boost in the photogenerated current of $42.7 \mathrm{~mA} \mathrm{~cm}^{-2}$. The record efficiency recorded by Alta Devices with GaAs was obtained with a relatively thin film (a few micrometres, rather than a few hundred micrometres as in standard cells). This brings us to the discussion of what determines high efficiency. A solar cell operates at a voltage that maximizes the generated power, dictated by the short-circuit current, FF and $V_{\mathrm{OC}}$. Designing with a view to achieving higher efficiencies requires strategies for increasing these values ${ }^{38,39}$. Improving the first two parameters - shortcircuit current and FF - mainly involves device 'engineering', while obtaining the ultimate $V_{\mathrm{OC}}$ is dictated by the thermodynamics of solar energy conversion into electrical work. Within the Shockley-Queisser model, $V_{\mathrm{OC}}$ is limited as follows ${ }^{36}$ :

$$
V_{\mathrm{oc}}=\frac{E_{\mathrm{g}}}{q}\left(1-\frac{T}{T_{\text {sun }}}\right)-\frac{k T}{q}\left(\ln \frac{\Omega_{\mathrm{emit}}}{\Omega_{\mathrm{sun}}}+\ln \frac{4 n^{2}}{C}-\ln \mathrm{QE}\right)
$$

where $T$ and $T_{\text {sun }}$ are the temperatures of the cell and the sun, respectively, $\Omega_{\mathrm{emit}}$ and $\Omega_{\text {sun }}$ correspond to the solid angle of 
Table 1 | $J_{S C}, F F, V_{O C}$, area and efficiency of the best photovoltaic technologies compared with the standing nanowire configuration presented in this work.

\begin{tabular}{|c|c|c|c|c|c|}
\hline Technology & $J_{\mathrm{SC}}\left(\mathrm{mA} \mathrm{cm}{ }^{-2}\right)$ & FF (\%) & $V_{\text {oc }}(\mathrm{V})$ & Area $\left(\mathrm{cm}^{2}\right)$ & $\eta(\%)$ \\
\hline $\mathrm{c}-\mathrm{Si}$ & 42.7 & 82.8 (ref. 47) & 0.706 & 4 & 25.0 \\
\hline GaAs & 29.68 & 86.5 & 1.122 & 1 & 28.8 (ref. 48) \\
\hline Triple junction & 14.57 & 86.0 & 3.014 & 30 & 37.7 \\
\hline This work & 180 & 52 & 0.43 & $1.42 \times 10^{-9}$ & $40^{\star}$ \\
\hline
\end{tabular}

The low $V_{O C}$ and $F F$ values indicate the potential for improvement of the nanowire cell presented. See main text for discussion.

${ }^{*}$ Apparent efficiency calculated with the projected area of the cell.

emission and collection, $n$ is the refractive index of the material, and $\mathrm{QE}$ is the emission quantum efficiency. The first term is related to the Carnot efficiency, which reduces $V_{\text {OC }}$ by $\sim 5 \%$. The second term corresponds to the entropic losses occurring during work generation. The first entropy loss is due to non-reciprocity in the angle of light absorption and emission. Light-resonant structures such as nanowires can reduce the contribution of this term ${ }^{36}$. The second entropy loss takes account of the concentration factor, given by the refractive index and any external concentration: $V_{\mathrm{OC}}$ increases by implementing light-trapping strategies, with the additional benefit of increasing absorption close to the bandgap ${ }^{40,41}$. The last entropic term refers to non-radiative losses. It can be reduced to zero by increasing QE to 1. The impressive result for GaAs cells achieved by Alta Devices was obtained thanks to increasing the $\mathrm{QE}$ in a GaAs thin film to 1 . Our results provide a further path for higher-efficiency solar cells. Even though the electrical characteristics shown are not yet ideal, we observe a light concentration effect plus a significant increase in absorption rate close to the bandgap, similar to that proposed in ref. 36. These two effects are such that nanowire structures can reduce the entropy in the conversion of solar energy into electrical work, thereby providing a path for increasing the efficiency of solar cells. It is also important to note that the unexpected increase in the absorption cross-section enables nanowires to be further separated from one another, resulting in major cost reductions for the final device. Our experiments indicate that a good interwire distance would be $\sim 1.2 \mu \mathrm{m}$. A nanowire solar cell consisting of nanowires similar to the device shown in Fig. 1, positioned in a hexagonal array with a pitch of $1 \mu \mathrm{m}$ (optical cross-section with a diameter of $\sim 1.2 \mu \mathrm{m}$ ), would have an optical FF of 1 , and it would only use a material volume equivalent to that of a $546 \mathrm{~nm}$ film and exhibit a conversion efficiency of $4.6 \%$. By using devices with a smaller area (see left branch in Fig. 2a,b), one could further reduce the amount of material used by a factor of up to 72 . By improving the electrical characteristics of the $\mathrm{p}-\mathrm{n}$ junction, higher efficiencies could be obtained. By considering an effective light concentration of only 15 , an array of GaAs nanowires with ideal characteristics would exhibit an efficiency of $33.4 \%$, thereby overcoming the Shockley-Queisser limit for planar GaAs solar cells illuminated by 1.5 AM radiation, according to the discussion presented in the foregoing text ${ }^{34,42}$. Even higher efficiencies could be achieved if the device design were tailored for higher light concentrations and QE. Note that axial junctions, which have the same junction area as the projected area, would obtain the full benefit from such a concentrator effect, and it would be possible to directly compare the performance of GaAs nanowire solar cells under 1 sun with planar GaAs cells under 10 suns. We demonstrate here that single-nanowire devices generate severalfold higher power than their projected areas suggest when they are standing upright, a configuration that also minimizes their footprint. It should be noted that if one were to build a single-nanowire solar cell, then a flat-lying nanowire would exhibit $\sim 15$ times lower power density compared to the standing nanowire device due to the light concentration effect. This enhancement in energy conversion at the nanoscale will make standing nanowires useful as energy harvesters, with minimum footprint, for feeding other devices on the same chip. This is already the case for nanowire-based $\mathrm{p}-\mathrm{n}$ junctions with non-ideal characteristics such as the one demonstrated in this work. Last, but not least, the improvement in photon collection renders them (in general) ideal for use as photodetectors.

In conclusion, we have observed a remarkable boost in absorption in single-nanowire solar cells that is related to the vertical configuration of the nanowires and to a resonant increase in the absorption cross-section. These results open a new route to thirdgeneration solar cells, local energy harvesters on nanoscale devices and photon detectors.

\section{Methods}

Nanowire growth. Nanowires were grown on oxidized $\mathrm{Si}(111)$ with $100 \mathrm{~nm}$ apertures using a self-catalysed method. The gallium nominal growth rate was $900 \mathrm{~nm} \mathrm{~h}^{-1}$, the substrate temperature was $630^{\circ} \mathrm{C}$ and the $\mathrm{V} / \mathrm{III}$ ratio was 4 (refs $43,44)$. The p-doping of the core was achieved by adding a flux of beryllium during axial growth ${ }^{45}$. Cores were annealed for $10 \mathrm{~min}$ at $630^{\circ} \mathrm{C}$. The shell was obtained at $465{ }^{\circ} \mathrm{C}$, a growth rate of $300 \mathrm{~nm} \mathrm{~h}^{-1}$ and with a $\mathrm{v} /$ III ratio of 50 . The n-type doping was obtained by adding silicon to the growth step ${ }^{46}$.

Device fabrication and characterization. SU-8 was spun onto the substrate at 4,000 r.p.m. for $45 \mathrm{~s}$ and cured with $1 \mathrm{~min}$ ultraviolet light and $3 \mathrm{~min}$ on a hotplate at $185{ }^{\circ} \mathrm{C}$. An etch-back with a 1-3 min oxygen plasma etch was then performed to free the nanowire tip. The top contact was defined by electron-beam lithography followed by evaporation of indium tin oxide (ITO) (for more details see Supplementary Section S1). The bottom contact was obtained by silver gluing to the back-side of the wafer. Current-voltage characteristics were measured in the dark and under $1.5 \mathrm{G}$ conditions with a standard solar simulator (LOT - Oriel $150 \mathrm{~W}$ xenon lamp) with a 1-inch beam diameter and an AM 1.5G filter. A photocurrent map of the devices was collected by scanning the contacted sample under the laser spot focused with a $\times 63$ and $0.75 \mathrm{NA}$ lens.

FDTD method simulations. The absorption of standing $2.5 \mu \mathrm{m}$ GaAs nanowires of different diameters, standing on silicon and surrounded by SU-8, was calculated by solving Maxwell equations in three dimensions for an incident plane-wave radiation normal to the substrate. The wave equation was solved in the time domain according to refs 30 and 27.

Received 6 November 2012; accepted 28 January 2013; published online 24 March 2013

\section{References}

1. Tian, B. Z. et al. Coaxial silicon nanowires as solar cells and nanoelectronic power sources. Nature 449, 885-888 (2007).

2. Law, M., Greene, L. E., Johnson, J. C., Saykally, R. \& Yang, P. D. Nanowire dye-sensitized solar cells. Nature Mater. 4, 455-459 (2005).

3. Kayes, B. M., Atwater, H. A. \& Lewis, N. S. Comparison of the device physics principles of planar and radial $p-n$ junction nanorod solar cells. J. Appl. Phys. 97, 114302 (2005).

4. Spurgeon, J. M. et al. Flexible, polymer-supported, Si wire array photoelectrodes. Adv. Mater. 22, 3277-3281 (2010)

5. Fan, Z. et al. Three-dimensional nanopillar-array photovoltaics on low-cost and flexible substrates. Nature Mater. 8, 648-653 (2009).

6. Kempa, T. J. et al. Single and tandem axial $p-i-n$ nanowire photovoltaic devices. Nano Lett. 8, 3456-3460 (2008).

7. Chuang, L. C. et al. Critical diameter for III-V nanowires grown on lattice-mismatched substrates. Appl. Phys. Lett. 90, 043115 (2007).

8. Glas, F. Critical dimensions for the plastic relaxation of strained axial heterostructures in free-standing nanowires. Phys. Rev. B 74, 121302 (2006).

9. Tsakalakos, L. et al. Silicon nanowire solar cells. Appl. Phys. Lett. 91, 233117 (2007)

10. Goto, H. et al. Growth of core-shell InP nanowires for photovoltaic application by selective-area metal organic vapor phase epitaxy. Appl. Phys. Express 2, 035004 (2009). 
11. Garnett, E. C. \& Yang, P. Silicon nanowire radial p-n junction solar cells. J. Am. Chem. Soc. 130, 9224-9225 (2008).

12. Wallentin, J. et al. InP nanowire array solar cells achieving $13.8 \%$ efficiency by exceeding the ray optics limit. Science 339, 1057-1060 (2013).

13. Tian, B. Z. \& Lieber, C. M. Design, synthesis, and characterization of novel nanowire structures for photovoltaics and intracellular probes. Pure Appl. Chem. 83, 2153-2169 (2011).

14. Dalmau-Mallorqui, A., Epple, F. M., Fan, D., Demichel, O. \& Fontcuberta i Morral, A. Effect of the pn junction engineering on Si microwire-array solar cells. Phys. Status Solidi 209, 1588-1591 (2012).

15. Tsakalakos, L. et al. Strong broadband optical absorption in silicon nanowire films. J. Nanophot. 1, 013552 (2007).

16. Zhu, J. et al. Optical absorption enhancement in amorphous silicon nanowire and nanocone arrays. Nano Lett. 9, 279-282 (2009).

17. Mariani, G. et al. Patterned radial GaAs nanopillar solar cells. Nano Lett. 11, 2490-2494 (2011).

18. Muskens, O. L., Gomez-Rivas, J., Algra, R. E., Bakkers, E. P. A. M. \& Lagendijk, A. Design of light scattering in nanowire materials for photovoltaic applications. Nano Lett. 8, 2638-2642 (2008).

19. Kosten, E. D., Warren, E. L. \& Atwater H. A. Ray optical light trapping in silicon microwires: exceeding the $2 n^{2}$ intensity limit. Opt. Express 19, 3316-3331 (2011).

20. Callahan, D. M., Munday, J. N. \& Atwater H. A. Solar cell light trapping beyond the ray optic limit. Nano Lett. 12, 214-218 (2012).

21. Kelzenberg, M. D. et al. Enhanced absorption and carrier collection in Si wire arrays for photovoltaic applications. Nature Mater. 9, 239-244 (2010).

22. Diedenhofen, S. et al. Strong geometrical dependence of the absorption of light in arrays of semiconductor nanowires. ACS Nano 5, 2316-2323 (2011).

23. Kwanyong, S. et al. Multicolored vertical silicon nanowires. Nano Lett. 11, 1851-1856 (2011)

24. Van Vugt, L. L., Zhang, B., Piccione, B., Spector, A. A. \& Agarwal, R. Sizedependent waveguide dispersion in nanowire optical cavities: slowed light and dispersionless guiding. Nano Lett. 9, 1684-1688 (2009).

25. Cao, L. et al. Engineering light absorption in semiconductor nanowire devices. Nature Mater. 8, 643-647 (2009).

26. Brönstrup, G. et al. A precise optical determination of nanoscale diameters of semiconductor nanowires. Nanotechnology 22, 385201 (2011).

27. Heiss, M. \& Fontcuberta i Morral A. Fundamental limits in the external quantum efficiency of single nanowire solar cells. Appl. Phys. Lett. 99, 263102 (2011).

28. Kempa, T. et al. Coaxial multishell nanowires with high-quality electronic interfaces and tunable optical cavities for ultrathin photovoltaics. Proc. Natl Acad. Sci. USA 109, 1407-1412 (2011).

29. Nelson, J. The Physics of Solar Cells (Imperial College, 2003).

30. Oskooi, A. F. et al. MEEP: a flexible free-software package for electromagnetic simulations by the FDTD method. Comp. Phys. Comm. 181, 687-702 (2010).

31. Kupec, J. \& Witzigmann, B. Dispersion, wave propagation and efficiency analysis of nanowire solar cells. Opt. Express 17, 10399-10410 (2009).

32. Cao, L. Y. et al. Semiconductor nanowire optical antenna solar absorbers. Nano Lett. 10, 439-445 (2010).

33. Leatherdale, C. A., Woo, W. K., Mikulec, F. V. \& Bawendi, M. G. On the absorption cross section of CdSe nanocrystal quantum dots. J. Phys. Chem. B 106, 7619-7622 (2002).

34. Henry, C. H. Limiting efficiencies of ideal single and multiple energy gap terrestrial solar cells. J. Appl. Phys. 51, 4494-4500 (1980).

35. Araújo, G. L. \& Marti, A. Absolute limiting efficiencies for photovoltaic energyconversion. Solar Ener. Mater. Solar Cells 33, 213-240 (1994).
36. Polman, A. \& Atwater H. A. Photonic design principles for ultrahigh-efficiency photovoltaics. Nature Mater. 11, 174-177 (2012).

37. Kempa, T. et al. Coaxial multishell nanowires with high-quality electronic interfaces and tunable optical cavities for ultrathin photovoltaics. Proc. Natl Acad. Sci. USA 109, 1407-1412 (2012).

38. Campbell, P. \& Green, M. A. The limiting efficiency of silicon solar cells under concentrated sunlight. IEEE Trans. Electron. Device Lett. 33, 234-239 (1986).

39. Nelson, J. The Physics of Solar Cells (Imperial College Press, 2003).

40. Luque, A. The confinement of light in solar cells. Solar Ener. Mater. 23, 152-163 (1991).

41. Yablonovitch, E. \& Cody, G. D. Intensity enhancement in textured optical sheets for solar cells. IEEE Trans. Electron. Device Lett. 29, 300-305 (1982).

42. Shockley, W. \& Queisser H. J. Detailed balance limit of efficiency of $p-n$ junction solar cells. J. Appl. Phys. 32, 510-519 (1961).

43. Uccelli, E. et al. Three-dimensional twinning of self-catalyzed GaAs nanowires on Si substrates. Nano Lett. 11, 3827-3832 (2011).

44. Krogstrup, P. et al. Structural phase control in self-catalyzed growth of GaAs nanowires on silicon (111). Nano Lett. 10, 4475-4482 (2010).

45. Casadei, A. et al. Doping incorporation paths in catalyst-free Be-doped GaAs nanowires. Appl. Phys. Lett. 102, 013117 (2013).

46. Colombo, C., Heiß, M., Graetzel, M. \& Fontcuberta i Morral, A. Gallium arsenide $\mathrm{p}-\mathrm{i}-\mathrm{n}$ radial structures for photovoltaic applications. Appl. Phys. Lett. 94, 173108 (2009).

47. Zhao, J. et al. $19.8 \%$ efficient 'honeycomb' textured multicrystalline and $24.4 \%$ monocrystalline silicon solar cells. Appl. Phys. Lett. 73, 1991-1993 (1998).

48. Green, M. A., Emery, K., Hishikawa, Y., Warta, W. \& Dunlop, E. D. Solar cell efficiency tables (version 41). Prog. Photovolt. 21, 1-11 (2013).

\section{Acknowledgements}

This research was funded by the ERC starting grant UpCon and by SNF (project nos 137648, 143908) and NCCR-QSIT. A.F.i.M. thanks STI for the 2011 end-of-year fund for MiBoots robots used in the scanning photocurrent experiment. A.F.i.M. and M.H. thank A. Dalmau-Mallorqui and F.M. Epple for experimental support. The authors also thank C.B. Sørensen and M.H. Madsen for assistance with MBE growth. This work was supported by the Danish National Advanced Technology Foundation (project 022-2009-1), a University of Copenhagen Center of Excellence, and by the UNIK Synthetic Biology project.

\section{Author contributions}

P.K. grew the nanowire $\mathrm{p}-\mathrm{n}$ junctions. H.I.J. performed $I-V$ characterization and fabricated the device, with help from J.V.H. and M.A. M.H. and O.D. performed the FDTD calculations. M.H. realized the photocurrent mappings and the external quantum efficiency measurements. A.F.i.M. and P.K. conceived and designed the experiments. A.F.i.M., J.N. and M.A. supervised the project. A.F.i.M., H.I.J., P.K. and M.H. composed the figures and wrote the manuscript. All authors discussed the results and commented on the manuscript.

\section{Additional information}

Supplementary information is available in the online version of the paper. Reprints and permissions information is available online at www.nature.com/reprints. Correspondence and requests for materials should be addressed to P.K. and A.F.i.M.

\section{Competing financial interests}

The authors declare no competing financial interests. 